\title{
Erratum to: Membrane Environment Imposes Unique Selection Pressures on Transmembrane Domains of G Protein-Coupled Receptors
}

\author{
Stephanie J. Spielman • Claus O. Wilke
}

Published online: 28 February 2013

(c) Springer Science+Business Media New York 2013

\section{Erratum to: J Mol Evol}

DOI 10.1007/s00239-012-9538-8

Error notified in the caption of Fig. 3 of published article, Fig. 3 incorrectly labels red points as TM and blue points as EM. Instead, the red points represent extramembrane residues, and the blue points represent transmembrane residues. Further, the shift of the red EM points towards higher RSA values reflects their reduced burial by neighboring residues. Corrected caption for Fig. 3 is given below:

Fig. 3 Regression of the $\log (\mathrm{dN} / \mathrm{dS})$ against RSA and residue partition. Red points represent EM residues and blue points represent TM residues. The gene name with its PDB ID in parentheses is shown above each graph. Linear regressions show that partition has a highly significant effect for each protein shown here (see Table 1 for more details). Further, the EM points display a noticeable shift towards higher RSA values, reflecting the decreased burial by neighboring residues experienced in that domain

The online version of the original article can be found under doi:10.1007/s00239-012-9538-8.

S. J. Spielman $(\bowtie) \cdot$ C. O. Wilke

The University of Texas at Austin, Austin, TX 78731, USA

e-mail: stephanie.spielman@utexas.edu 